Reversible pulmonary hypertension and high-output heart failure triggered by pregnancy in a patient with congenital arteriovenous malformation:

\section{A case report}

\author{
Héctor Saavedra', Celina Toncel ${ }^{2}$, Vanessa Delgado ${ }^{3}$, \\ Orlando Borré ${ }^{4}$ and José Rojas-Suárez ${ }^{5}$
}

Obstetric Medicine 2019, Vol. 12(2) 97-99 (C) The Author(s) 2018 Article reuse guidelines: sagepub.com/journals-permissions DOI: $10.1 \mid 77 / 1753495 \times 18766975$ journals.sagepub.com/home/obm

(9SAGE

\begin{abstract}
Background: Arteriovenous malformations rarely cause congestive heart failure. Pregnancy may in theory trigger heart failure associated with congenital arteriovenous malformations leading to secondary pulmonary hypertension, but no cases have been reported proving that condition. Methods and results: We report a 23-year-old pregnant woman at $36+5$ weeks of gestation requiring urgent medical care because of shortness of breath. High-output heart failure was suspected, and a congenital arteriovenous malformation on the right scapular region was considered as the possible origin. The patient required urgent caesarean delivery because of ongoing cardiac failure, which improved soon after delivery. Postpartum angiography of the right subclavian artery revealed an arteriovenous malformation on the deltoid region with venous drainage through the subclavian vein and increased flow to the superior cava vein and right atrium.

Conclusion: A high index of suspicion of arteriovenous malformations should be maintained in pregnant women with cutaneous vascular malformation-like lesions, if symptoms of heart failure are present.
\end{abstract}

\title{
Keywords
}

Pregnancy, high cardiac output, arteriovenous malformations, heart failure

\section{Background}

Pregnancy involves a series of adaptive changes, including hormonal and cardiovascular changes. ${ }^{1}$ These increase cardiac output by 30 to $50 \%$, and also increase systolic volume and heart rate, ${ }^{2,3}$ in addition to a decrease in mean arterial pressure and peripheral vascular resistance. All these changes may lead to a clinical relapse in patients with history of heart disease or heart failure. ${ }^{4}$

Heart failure is an abnormality of the cardiac structure or function that means the heart is unable to deliver oxygen in accordance with the requirements of the tissues. ${ }^{5}$ This is commonly associated with low cardiac output but may also be associated with high cardiac output (i.e. high output heart failure) defined as having a cardiac output $>8 \mathrm{~L} / \mathrm{min}$ or cardiac index $>4.0 \mathrm{~L} / \mathrm{min} / \mathrm{m}^{2}{ }^{6}$ This condition is linked to multiple disorders, such as chronic anaemia, thyrotoxicosis, sepsis, obesity, hepatic diseases and pregnancy. ${ }^{7}$ A high output situation is often associated with ventricular dilatation and/or hypertrophy, persistent tachycardia and heart valve dysfunction, which may eventually trigger heart failure. ${ }^{7}$

Pregnancy and arteriovenous malformations (AVMs) are independent causes of high output heart failure. ${ }^{6}$ However, to our knowledge, there are no reports published in which these two conditions coexist and cause pulmonary hypertension and heart failure. We describe a rare case of a pregnant patient with a reversible high output heart failure secondary to an AVM, which improved after delivery.

\section{Case report}

A 23-year-old woman, $36+5$ weeks into her second pregnancy, came to our hospital complaining of exertional dyspnea and orthopnea for three months. She described having a congenital cutaneous lesion on her right scapular region which had significantly increased in size during her previous pregnancy corresponding to the onset of the described symptoms. On physical examination the most prominent findings were an increased jugular venous pressure, a 2/6 systolic

\footnotetext{
'Department of Gynecology and Obstetrics, Hospital Divina Misericordia, Magangué, Colombia

${ }^{2}$ Fundación Cardiovascular de Colombia, Bucaramanga, Colombia

${ }^{3}$ Department of Obstetrics and Gynecology, Universidad Autónoma de Bucaramanga, Bucaramanga, Colombia

${ }^{4}$ Department of Gynecology and Obstetrics, Universidad de Cartagena, Cartagena, Colombia

${ }^{5}$ Intensive Care and Obstetric Research Group (GRIClO), Universidad de Cartagena, Cartagena, Colombia
}

Corresponding author:

Jose Rojas-Suarez, Gestion Salud Clinic, Cra 8I Calle 15, Cartagena, Colombia.

Email: joseantonio.rojas.suarez@gmail.com 


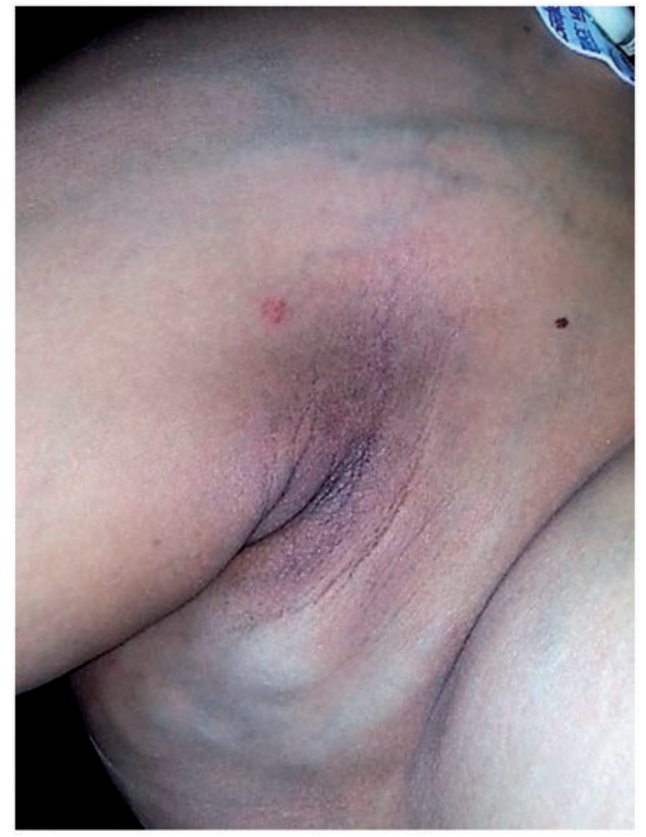

Figure I. Arteriovenous malformation presenting as a vascular patch and venous dilatation on right scapular region extending to the axillary region.

murmur at the left upper sternal border and a cutaneous lesion on the right scapular region of $12 \times 10 \mathrm{~cm}$, which was bright red, raised and warm, with a palpable thrill a thrill and venous dilation extending to the axillary region (Figure 1). Physical examination was otherwise unremarkable, including fetal assessment. Chest radiography showed cardiomegaly and transthoracic echocardiogram revealed a normal ejection fraction $(66 \%)$, resting cardiac index beyond the normal range ( 2.5 to $4 \mathrm{~L} / \mathrm{min} / \mathrm{m}^{2}$ ), mild bi-atrial and right ventricular dilation and significant tricuspid regurgitation with indirect signs of mild pulmonary hypertension (estimated pulmonary artery systolic pressure of $38 \mathrm{mmHg}$ ). An abdominal ultrasound showed normal kidneys, with large dilated veins on both sides. A clinical diagnosis of high output heart failure was suspected, and a congenital arteriovenous fistula on the right scapular region was considered as the possible source. A medical board considered termination of pregnancy and proposed postpartum angiography of the right subclavian artery. The patient was transferred to the intensive care unit for monitoring. She underwent caesarean section; a giant uterine vascular dilation was evident during surgery. A $3100 \mathrm{~g}$ healthy male newborn was delivered with no post-surgical complications. Postpartum angiography of her right subclavian artery showed the presence of an AVM located on the deltoid region with main branches of the right subclavian artery and venous drainage through the subclavian vein, with increased flow to the superior vena cava and right atrium (Figure 2). The patient improved soon after delivery. An echocardiogram performed two weeks postpartum showed a dramatic improvement of right cardiac filling pressures and resolution of the pulmonary hypertension. Delayed surgical obliteration by selective arterial embolization four weeks postpartum was suggested. Unfortunately, the patient did not return for follow-up.

\section{Discussion}

AVMs may lead to cardiac structural changes, including increased heart rate as a compensatory mechanism and decreased systemic

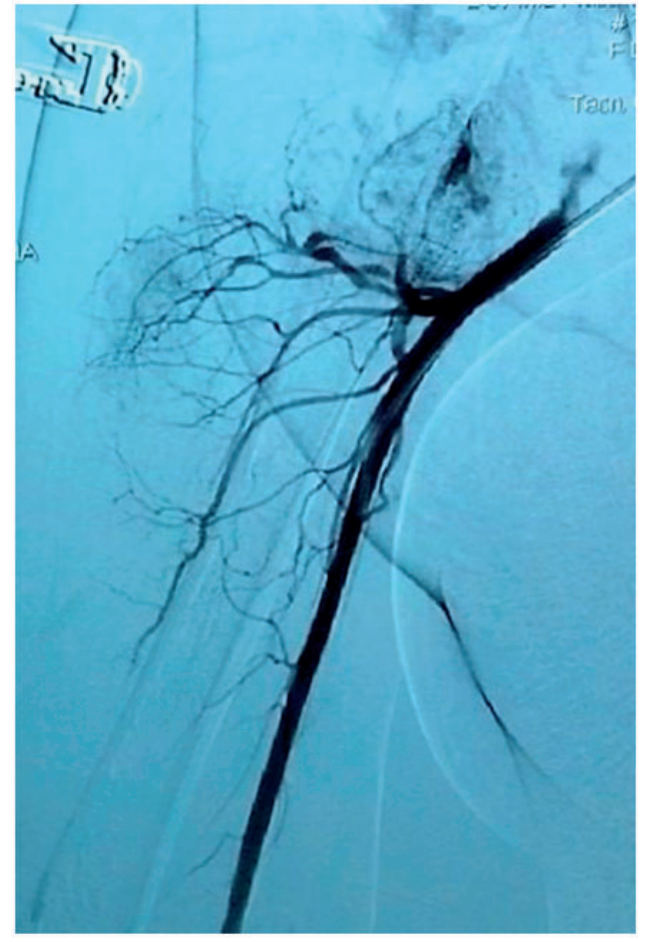

Figure 2. Right subclavian angiography showing an arteriovenous malformation with right subclavian afferents and venous drainage through subclavian vein.

vascular resistance with activation of the sympathetic nervous system, which eventually may lead to high output heart failure. ${ }^{7}$

The differential diagnosis of congenital vascular abnormalities is often necessary. Generally, these affect the skin and are evident at birth or within the first weeks of life. Although haemangiomas and AVMs may be similar, some differences often arise during disease progression and treatment. Haemangiomas usually appear during the first weeks or months after birth and retract spontaneously over time, whereas AVMs are present at birth and tend to grow throughout life. ${ }^{8}$

AVMs refer to a group of congenital fast-flow vascular malformations composed of abnormal arteries, veins and capillaries, with direct arteriovenous communications that provoke blood shunting. ${ }^{9}$ The most frequent location of AVMs is intracranial followed by head, neck, limbs, trunk and viscera. As the malformation develops, the degree of ectasia increases and becomes apparent, with venous dilatation and arterial hypertrophy. A decrease in systemic vascular resistance may produce cardiac symptoms which can lead to heart failure due to an arteriovenous shunt. ${ }^{7}$ In our patient, the diagnosis was considered due to the persistence of a vascular patch and was confirmed postpartum on angiography.

To see pregnant patients with pulmonary hypertension and/or heart failure is not uncommon, despite the fact that these are entities that contraindicate pregnancy due their risk of bad obstetric and perinatal outcomes. ${ }^{10,11}$ However, to the best of our knowledge, the coexistence of these entities (AVM, pregnancy, pulmonary hypertension and heart failure), leading to deterioration of cardiovascular condition as the pregnancy progresses, has never been reported. There is little evidence about the mode of delivery in patients with heart failure. However, a recent systematic review of different modes of delivery in patients with adult congenital heart disease found no increase in maternal or neonatal complications in patients with a NYHA class I and II in labour. On the other hand, women with a 
functional class NYHA III and IV at labour had higher complication rates with adverse maternal and fetal outcomes and longer intensive care and hospital length of stay. ${ }^{12}$ Thus, vaginal delivery under these conditions might be a factor that worsened the cardiac condition of our patient, in addition to the common presence of anaemia. All of these are well-known risk factors associated with haemodynamic instability and maternal-fetal adverse outcomes. ${ }^{7}$

In these situations, no strong recommendations emerge about a safe mode of delivery and it is often difficult to achieve a consensus on the management because of the exceptional presentation and the lack of strong evidence. Thus, management must be multidisciplinary, including gynaecologists, interventional radiologists, anaesthesiologists, intensivists and specialists in maternal-fetal medicine, in order to provide the best technical, clinical and scientific knowledge, and decrease the risk of adverse outcomes.

\section{Conclusions}

Pregnancy carries an increased risk of progression of cardiovascular diseases; hence, it is necessary that both medical staff and the patient stay well informed. A high index of suspicion of AVMs should be considered in women with malformation-like lesions if symptoms of heart failure are present during pregnancy, requiring in many cases antenatal counselling and a multidisciplinary team approach before, during and after childbirth.

\section{Acknowledgements}

The authors thank Aura Gonzalez, for her participation in technical editing of the manuscript.

\section{Declaration of conflicting interests}

The author(s) declared no potential conflicts of interest with respect to the research, authorship, and/or publication of this article.

\section{Funding}

The author(s) received no financial support for the research, authorship, and/or publication of this article.

\section{Ethical approval}

Written informed consent was obtained for this case report. Ethics board approval was not required in accordance with the policy of our hospital for case report publication.

\section{Guarantor}

JAR-S.

\section{Contributorship}

Substantial contributions to the conception or design of this paper; or the acquisition, analysis, or interpretation of data for the manuscript: J.A. Rojas-Suarez, H. Saavedra; Drafting the work or revising it critically for important intellectual content: J.A. Rojas-Suarez., H. Saavedra., C. Toncel., V. Delgado., O.R. Borré; Final approval of the version to be published: J.A. Rojas-Suarez, H. Saavedra; Agreement in all aspects of the work, ensuring that questions related to the accuracy or integrity of any part of the work were appropriately investigated and resolved: J.A. Rojas-Suarez, H. Saavedra, C. Toncel, V. Delgado, O.R. Borré.

\section{References}

1. Ouzounian JG and Elkayam U. Physiologic changes during normal pregnancy and delivery. Cardiol Clin 2012; 30: 317-329.

2. Christianson RE. Studies on blood pressure during pregnancy. I. Influence of parity and age. Am J Obstet Gynecol 1976; 125: 509-513.

3. Chesnutt AN. Physiology of normal pregnancy. Crit Care Clin 2004; 20: 609-615. doi:10.1016/j.ccc.2004.06.001.

4. Greutmann M and Pieper PG. Pregnancy in women with congenital heart disease. Eur Heart J 2015; 36: 2491-2499.

5. Tanai E and Frantz S. Pathophysiology of heart failure. In: Comprehensive physiology (vol. 6). Hoboken, NJ: John Wiley \& Sons, Inc., 2015, pp.187-214.

6. Wasse H and Singapuri MS. High-output heart failure: how to define it, when to treat it, and how to treat it. Semin Nephrol 2012; 32: 551-557.

7. Mehta PA and Dubrey SW. High output heart failure. QJM 2009; 102: 235-241.

8. Clemens RK, Pfammatter T, Meier TO, et al. Vascular malformations revisited. VASA 2015; 44: 5-22.

9. Ernemann U, Kramer U, Miller S, et al. Current concepts in the classification, diagnosis and treatment of vascular anomalies. Eur J Radiol 2010; 75: 2-11.

10. Aksu U, Topcu S and Tanboga IH. A lethal coexistence: pulmonary hypertension with pregnancy. Int $J$ Cardiol 2015; 195: 27-28.

11. Ladouceur M, Benoit L, Radojevic J, et al. Pregnancy outcomes in patients with pulmonary arterial hypertension associated with congenital heart disease. Heart 2017; 103: 287-292.

12. Asfour V, Murphy MO and Attia R. Is vaginal delivery or caesarean section the safer mode of delivery in patients with adult congenital heart disease? Interact Cardiovasc Thorac Surg 2013; 17: 144-150. 